\title{
In vivo detection of desmoplastic reaction using endocytoscopy: A new diagnostic marker of submucosal or more extensive invasion in colorectal carcinoma
}

\author{
YUUSAKU SUGIHARA ${ }^{1,2}$, SHIN-EI KUDO ${ }^{1}$, HIDEYUKI MIYACHI ${ }^{1}$, KUNIHIKO WAKAMURA $^{1}$, YUICHI MORI ${ }^{1}$, \\ MASASHI MISAWA ${ }^{1}$, TOMOKAZU HISAYUKI ${ }^{1}$, TOYOKI KUDO ${ }^{1}$, TAKEMASA HAYASHI ${ }^{1}$, \\ SHIGEHARU HAMATANI ${ }^{3}$, SHOGO OKOSHI ${ }^{4}$ and HIROYUKI OKADA ${ }^{2}$
}

\begin{abstract}
${ }^{1}$ Digestive Disease Center, Showa University Northern Yokohama Hospital, Yokohama, Kanagawa 224-0032;
${ }^{2}$ Department of Gastroenterology and Hepatology, Okayama University Graduate School of Medicine, Dentistry and Pharmaceutical Sciences, Okayama 700-8558; ${ }^{3}$ Department of Pathology, The Jikei University School of Medicine, Tokyo 105-8461; ${ }^{4}$ Department of Internal Medicine, School of Life Dentistry at Niigata,
\end{abstract}

The Nippon Dental University, Niigata 951-1500, Japan

Received October 11, 2016; Accepted November 29, 2016

DOI: $10.3892 / \mathrm{mco} .2017 .1138$

\begin{abstract}
The pathological determination of desmoplastic reaction (DR) in colorectal carcinoma is useful for predicting extensive submucosal invasion. The aim of the present study was to determine the usefulness of endocytoscopy (EC) in detecting DR. A total of 72 cases of colorectal cancer with submucosal invasion (EC classification, EC3b) were evaluated. The utility of fine granular structure (FGS) observed via EC for the prediction of the presence of DR in the most superficial tumor layers was assessed. Of the 72 lesions, 26 were positive for FGS, and the majority of these lesions $(23 / 26,88.5 \%)$ exhibited a DR, indicating a significant association. The overall accuracy of the identification of FGS via EC that was predictive of a DR was $87.3 \%$. The presence of FGS detected by EC was significantly associated with the presence of a DR, suggesting the clinical usefulness of EC in planning treatment for colon cancer with submucosal invasion.
\end{abstract}

Correspondence to: Dr Yuusaku Sugihara, Department of Gastroenterology and Hepatology, Okayama University Graduate School of Medicine, Dentistry and Pharmaceutical Sciences, 2-5-1 Shikata-cho, Kita-ku, Okayama 700-8558, Japan

E-mail: y.sugi117@gmail.com

Abbreviations: DR, desmoplastic reaction; EC, endocytoscopy; FGS, fine granular structure; NPV, negative predictive value; PPV, positive predictive value; SM, submucosa

Key words: endoscopy, colonoscopy, desmoplastic reaction, colorectal neoplasms, endoscopic examination

\section{Introduction}

The development of the magnifying scope is associated with major benefits regarding diagnosis of early colon cancer by enabling in vivo observation of the pit patterns on the tumor surface $(1,2)$. Recently, the invention of ultramagnifying endocytoscopy (EC) has enabled observation at a 400 -fold magnification. As the EC findings correspond well with the pathological findings, it is possible to detect living tumor cells and micro vessels in vivo (3-5) and obtain a pathological image by simply applying the scope to the target mucosa during an endoscopic examination, which serves as a mode of on-site 'optical biopsy' (6,7).

However, a desmoplastic reaction (DR), characterized by stromal myofibroblast infiltration of the outermost layers of the tumor (8), is considered to be a response to the invasion of carcinoma cells beyond the muscularis mucosae into the submucosa (SM) or deeper layers (9). Detection of a DR may be useful for predicting massive SM invasion by colorectal carcinoma (10). The fibrotic deposition of the cancer cells is caused by stromal cells, principally myofibroblasts and activated fibroblasts (11). These fibroblasts synthesize collagen, namely types I, III and IV, and proteoglycans that constitute the bulk of the DR. These collagens accumulate around the tumor and directly affect the growth and invasion of cancer cells. This process is considered to be a host defense mechanism intended to confine the developing tumor. However, DR has been associated with tumor progression and poor prognosis in colorectal carcinoma (12).

The non-structural pit pattern $\mathrm{V}$ (type $\mathrm{V}_{\mathrm{N}}$ pit pattern), which lacks a superficial microstructure, has been associated with a superficial DR that reflects deeply invasive submucosal components (an exposing DR) (13). However, to the best of our knowledge, in vivo assessment of DR using EC has not been reported to date.

EC enables the visualization of cellular membrane structures and nuclear morphology. Therefore, the aim of the present 


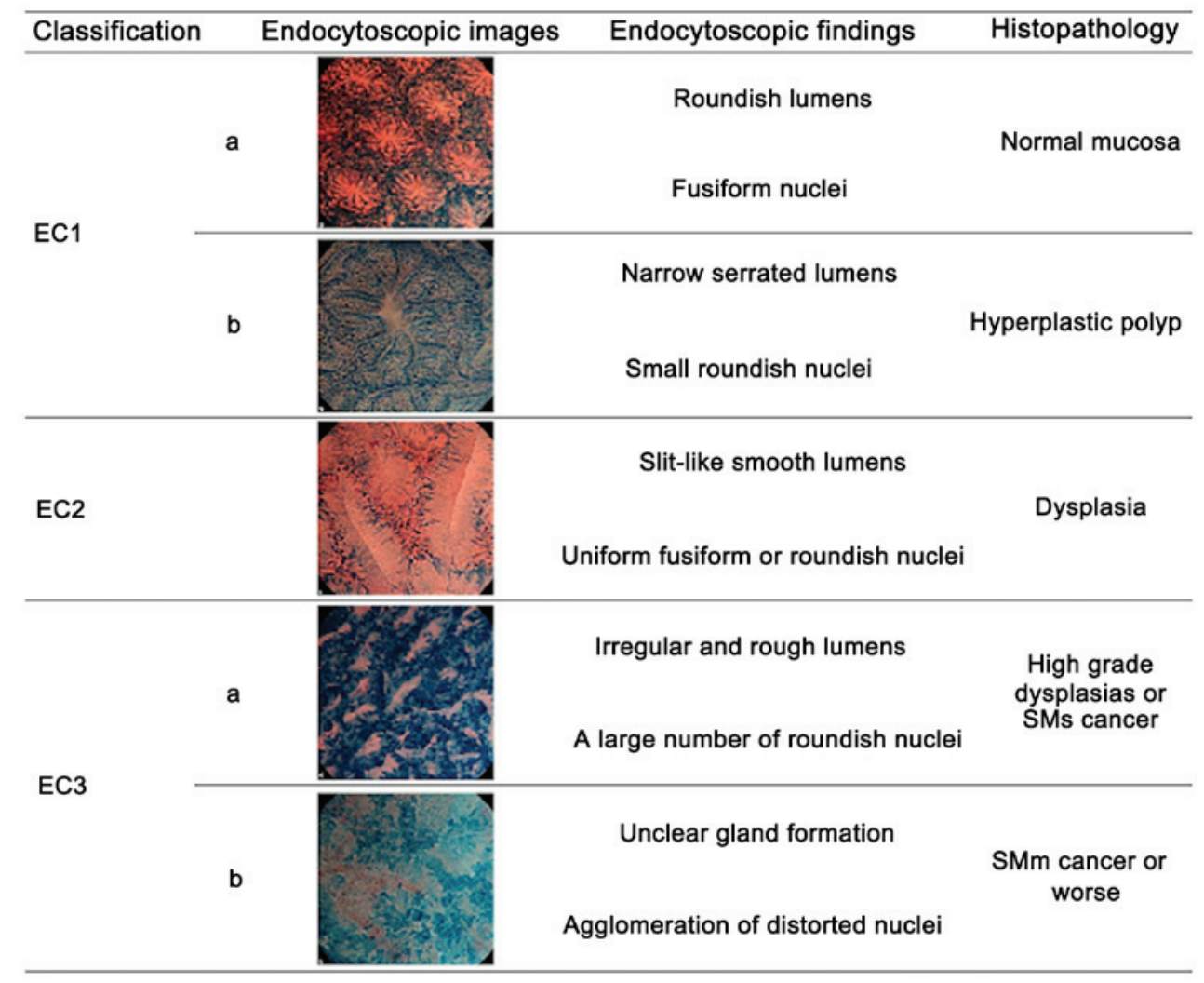

Figure 1. Definitions of endocytoscopic classification. ECla: Roundish lumina and regular pattern of uniformly sized fusiform nuclei. EClb: Narrow serrated lumina and dense pattern of small roundish nodules. EC2: Slit-like smooth lumina and regular pattern of fusiform or roundish nuclei. EC3a: Irregular and rough lumina and a large number of roundish nuclei strongly stained with methylene blue. EC3b: Unclear gland formation and agglomeration of distorted nuclei strongly stained with methylene blue. EC, endocytoscopy; SMs, slightly invasive submucosal cancer; SMm, massively invasive submucosal cancer.

study was to identify specific EC findings that may be used to detect DR histopathologically. Furthermore, we examined whether EC enabled detection of DRs indicative of invasive tumors. To the best of our knowledge, no previous study has described EC imaging findings with regards to superficial DRs in colorectal cancer to date.

\section{Materials and methods}

Patients and clinical data. A total of 49,990 patients underwent colonoscopy at the Digestive Disease Center, Showa University Northern Yokohama Hospital (Yokohama, Japan) between May, 2005 and August, 2013, and a total of 17,331 lesions were detected. Patients with familial adenomatous polyposis, tumor-related inflammatory bowel disease or a history of chemotherapy were excluded. Of all included lesions, 502 were identified using EC, with 72 diagnosed as EC3b according to the EC classification (6), suggesting invasion of the SM or deeper layers (Fig. 1). All the lesions were treated with endoscopic mucosal resection/endoscopic submucosal dissection or surgical resection. This study was granted ethical approval by the local Ethics Review Committee (approval no. 1410-07) and informed consent was obtained from all the participants prior to enrollment in this clinical trial.

Evaluation of EC. All examinations were performed with an integrated-type endocytoscope (XCF-260EC1; Olympus Co.,
Tokyo, Japan). All endoscopic procedures were performed by any one of four experienced endoscopists (Y.S., Y.M., M.M. and H.M.) who have performed $>100$ EC procedures over a period of 2 years.

It was hypothesized that the presence of a fine granular structure (FGS) may be a reliable indicator of DR (Fig. 2). FGS identified via EC was defined according to the following criteria: i) Presence of distorted nuclei, indicative of cancer cells, around the FGS; and ii) increased granularity of the FGS nuclei compared with the surrounding cancer cells, indicative of myofibroblasts or inflammatory cells. To ensure objectivity of the FGS findings, independent external reviewers blinded to the patient information calculated the interobserver agreement index regarding the assessment of FGS on the basis of the EC histological evaluations.

Histological evaluation. Each endoscopically or surgically resected specimen was fixed in $10 \%$ formalin and embedded in paraffin wax. The tissue specimens were then sliced into 2-mm sections and stained with hematoxylin and eosin. All the specimens were routinely evaluated to establish a pathological diagnosis. An experienced gastrointestinal pathologist evaluated all the specimens and assessed the presence of DR in the superficial layers of the tumor according to the following criteria reported by Kimura et al (14): i) Presence of carcinoma is required for the detection of an exposing DR; and ii) a DR involves an area of collagen fiber accumulation, myofibroblast proliferation and inflammatory infiltration. 

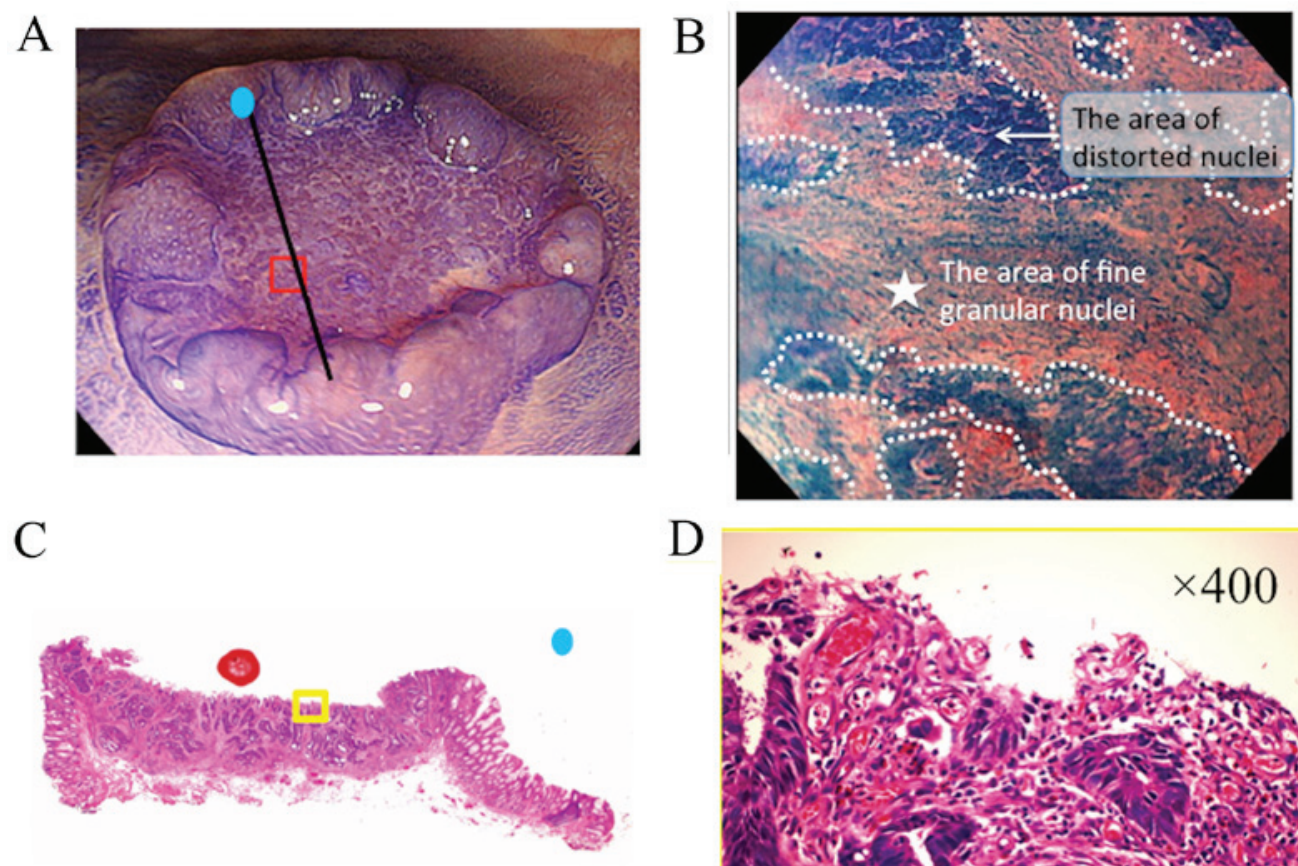

$\mathrm{D}$

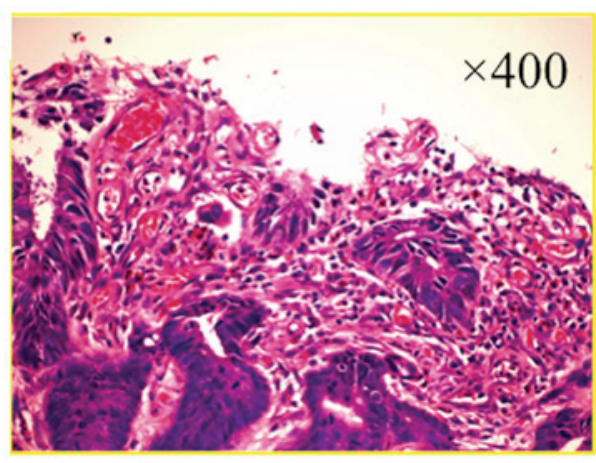

Figure 2. Correlation of FGS and histological findings in sigmoid colon carcinoma. (A) Depressed-type early sigmoid colon carcinoma stained with crystal violet. (B) EC image. The area denoted by the white star indicates FGS. FGS was defined according to the following criteria: i) Distorted nuclei adjacent to the FGS (white arrow) and ii) more granular nuclei in the FGS compared with the adjacent distorted nuclei. (C) Representative example of a DR in a hematoxylin and eosin-stained specimen. (D) The yellow square outlines a 400-fold magnified image. Inflammatory cells, but not cancer cells, are observed on the tumor surface. The DR is clearly demonstrated. The red square in (A) outlines a magnified view of the lesion on the EC image. The black bar represents the specimen section used for pathological examination in (C and D). FGS, fine granular structure; DR, desmoplastic reaction; EC, endocytoscopy.

Statistical analysis. All analyses were performed using STATA software, version 11.2 (StataCorp, College Station, TX, USA). The sensitivity, specificity, positive predictive value (PPV), negative predictive value (NPV) and overall accuracy of identifying DRs in the superficial layer of the tumors were estimated. P-values $<0.05$ were considered to indicate statistically significant differences.

Validation study for FGS. Interobserver agreement was assessed using $\mathrm{\kappa}$ statistics and interpreted as proposed by Landis and Koch (15). A $\kappa$ value of 0 demonstrated the absence of agreement; $<0.20$, slight agreement; $0.21-0.40$, fair agreement; 0.41-0.60; moderate agreement; 0.61-0.80, substantial agreement; and $>0.81$, almost perfect agreement.

\section{Results}

Patient characteristics. A total of 72 consecutive patients diagnosed with EC3b colorectal carcinoma by EC were enrolled in this study (median age, 65 years; range, $40-85$ years). Of the 72 patients, 46 were men. The clinical and pathological characteristics are summarized in Table I.

Comparison of tumor invasion depth between superficial exposing DRs and FGS. A comparison of the depth of tumor invasion among lesions with a superficial exposing DR and FGS detected via EC is shown in Table II. A close association with tumor invasion depth was observed for DR-positive and
Table I. Patient and tumor characteristics.

\begin{tabular}{lc}
\hline Characteristics & $\mathrm{n}(\%)$ \\
\hline Patients & $72(100.0)$ \\
Median age, years (range) & $65(40-85)$ \\
Median tumor size, mm (range) & $21(4-94)$ \\
Gender & \\
Male/female & $46(64.0) / 26(36.0)$ \\
Location & \\
Cecum & $2(2.8)$ \\
Ascending colon & $13(18.1)$ \\
Transverse colon & $5(6.9)$ \\
Descending colon & $3(4.2)$ \\
Sigmoid colon & $23(31.9)$ \\
Rectum & $27(37.5)$ \\
Morphology & \\
Pedunculated & $5(6.9)$ \\
Nonpedunculated & $67(93.1)$ \\
Histological appearance & \\
Well-/moderately differentiated & $52(72.2)$ \\
Poorly differentiated & $20(27.8)$ \\
\hline
\end{tabular}

FGS-positive findings. Of the 72 lesions, 26 were FGS-positive. As shown in Table III, the majority of these lesions (23/26; 
Table II. Comparison of tumor invasion depth between the superficial exposing DRs and FGS detected on endocytoscopy.

\begin{tabular}{lcccc}
\hline & \multicolumn{2}{c}{ Superficial exposing DR } & \multicolumn{2}{c}{ Endocytoscopy } \\
\cline { 2 - 4 } $\begin{array}{l}\text { Depth of } \\
\text { invasion }\end{array}$ & $\begin{array}{c}\text { Superficial } \\
\text { exposure }(+), \mathrm{n}(\%)\end{array}$ & $\begin{array}{c}\text { Superficial } \\
\text { exposure }(-), \mathrm{n}(\%) \\
(\mathrm{n}=30)\end{array}$ & $\begin{array}{c}\text { FGS }(+), \mathrm{n}(\%) \\
(\mathrm{n}=26)\end{array}$ \\
\hline M & $0(0.0)$ & $3(7.1)$ & $0(0.0)$ & $3(6.5)$ \\
SM & $23(76.7)$ & $34(80.1)$ & $19(73.1)$ & $39(84.8)$ \\
MP & $1(3.3)$ & $2(4.8)$ & $5(3.8)$ & $2(4.3)$ \\
SS & $5(16.7)$ & $1(2.4)$ & $19.2)$ & $7(15.2)$ \\
SE & $1(3.3)$ & $0(0.0)$ & $1(3.8)$ & $2(4.3)$ \\
\hline
\end{tabular}

DR, desmoplastic reaction; FGS, fine granular structure; SM, submucosa; MP, muscularis propria; SS, subserosa; SE, serosal exposure.

Table III. Correlation between the presence of FGS and superficial exposing DR.

\begin{tabular}{lrcc}
\hline & \multicolumn{2}{c}{ FGS } & \\
\cline { 2 - 3 } DR exposure & \multicolumn{1}{c}{ Positive } & Negative & Total \\
\hline Positive & $23(31.9)$ & $7(9.7)$ & $30(41.7)$ \\
Negative & $3(4.17)$ & $39(54.1)$ & $42(58.3)$ \\
Total & $26(36.1)$ & $46(63.9)$ & $72(100.0)$ \\
\hline
\end{tabular}

Values are presented as $\mathrm{n}(\%)$. DR, desmoplastic reaction; FGS, fine granular structure. $\mathrm{P}<0.0001, \kappa=0.708738$, standard deviation $=0.084723$.

88.5\%) exhibited an exposing DR detected by the presence of FGS on EC, which indicates a significant association. The overall accuracy, sensitivity, specificity, PPV and NPV for the utility of FGS on EC imaging for the detection of an exposing DR was $87.3,91.9,76.7,90.1$ and $80.2 \%$, respectively.

The mean $\mathrm{k}$ score for interobserver agreement between the two independent reviewers was 0.720 , indicating that the observers' evaluation of the FGS had substantial accuracy. The mean sensitivity, specificity, PPV and NPV for the detection of an FGS predictive of DR histopathology was 85.5, 73.6, 93.1 and $75.4 \%$, respectively.

\section{Discussion}

To the best of our knowledge, no previous study has reported EC imaging findings that may be used to detect a superficial exposing DR in colorectal cancer. In the present study, we observed that the presence of FGS on the EC image was a reliable marker of a DR, without the need for pathological examination. Thus, EC may have the potential to be a 'one-step' diagnostic tool for detecting superficial exposing DRs in colorectal cancer based on FGS findings.

The detection of DRs in pretreatment biopsy specimens has been reported to be useful for the prediction of early colorectal cancer invading the SM; therefore, DR status in a pathology report allows patients to be directed towards appropriate therapy, such as endoscopic mucosal resection/endoscopic submucosal dissection treatment or surgical resection (10).

Invasive carcinoma is characterized by the interruption of normal cellular compartments. The invasion field between pre-existing epithelial and stromal compartments is a critical interface in carcinogenesis. Stromal fibroblasts in a DR are critical in the development of digestive tract cancers (16). Accordingly, we hypothesized that there are several promising clinical implications of stromal fibroblast research for the prevention, diagnosis and management of digestive tract cancers. The presence of FGS detected via EC may be easily confirmed in one step, and this may significantly contribute to the future of this line of research.

A limitation of the present study was that the evaluation of FGS was subjective; however, an interobserver agreement index was obtained from multiple independent specialists. Although the obtained index value was significant (0.720), it is not adequate to provide sufficient objectiveness for the evaluation of the presence of FGS. Further studies are required to validate these findings and minimize interobserver variations. Prospective randomized studies, comparing the efficacy of identifying FGS by using EC with the histopathological assessment of DR as a diagnostic tool used for predicting treatment and patient outcomes after endoscopic or surgical resection may confirm the usefulness of EC. These future studies may improve the diagnosis of colorectal cancer and support the utility of 'one-step' diagnosis without the need for biopsy.

In conclusion, histological evaluation of biopsy specimens remains the standard approach for identifying DR. However, the presence of FGS identified on EC is likely to indicate an exposing DR. A DR is of vital importance to cancer cell migration and invasiveness. We consider that EC may improve the diagnosis of colorectal cancer and become the gold standard approach in the future, replacing the combination of conventional endoscopy and biopsy.

\section{Acknowledgements}

The authors express their gratitude to all the staff members at the Digestive Disease Center and the Department of Pathology, Showa University Northern Yokohama Hospital, for their excellent assistance. 


\section{References}

1. Kudo S, Hirota S, Nakajima T, Hosobe S, Kusaka H, Kobayashi T, Himori $\mathrm{M}$ and Yagyuu A: Colorectal tumors and pit pattern. J Clin Pathol 47: 880-885, 1994.

2. Kudo S, Rubio CA, Teixeira CR, Kashida H and Kogure E: Pit pattern in colorectal neoplasia: Endoscopic magnifying view. Endoscopy 33: 367-373, 2001.

3. Inoue H, Kazawa T, Sato Y, Satodate H, Sasajima K, Kudo SE and Shiokawa A: In vivo observation of living cancer cells in the esophagus, stomach, and colon using catheter-type contact endoscope, 'Endo-Cytoscopy system'. Gastrointest Endosc Clin N Am 14: 589-594 x-xi, 2004.

4. Sasajima K, Kudo SE, Inoue H, Takeuchi T, Kashida H, Hidaka E, Kawachi H, Sakashita M, Tanaka J and Shiokawa A: Real-time in vivo virtual histology of colorectal lesions when using the endocytoscopy system. Gastrointest Endosc 63: 1010-1017, 2006

5. Kudo SE, Misawa M, Wada Y, Nakamura H, Kataoka S, Maeda Y Toyoshima N, Hayashi S, Kutsukawa M, Oikawa H, et al: Endocytoscopic microvasculature evaluation is a reliable new diagnostic method for colorectal lesions (with video). Gastrointest Endosc 82: 912-923, 2015.

6. Mori Y, Kudo S, Ikehara N, Wakamura K, Wada Y, Kutsukawa M, Misawa M,Kudo T, Kobayashi Y, Miyachi H, et al: Comprehensive diagnostic ability of endocytoscopy compared with biopsy for colorectal neoplasms: A prospective randomized noninferiority trial. Endoscopy 45: 98-105, 2013.

7. Kudo SE, Wakamura K, Ikehara N, Mori Y, Inoue H and Hamatani S: Diagnosis of colorectal lesions with a novel endocytoscopic classification-a pilot study. Endoscopy 43: 869-875, 2011.

8. Martin M, Pujuguet P and Martin F: Role of stromal myofibroblasts infiltrating colon cancer in tumor invasion. Pathol Res Pract 192: 712-717, 1996.
9. Hirose M, Fukui H, Igarashi Y, Fujimori Y, Katake Y, Sekikawa A, Ichikawa K, Tomita S, Imura J, Ajioka Y, et al: Detection of desmoplastic reaction in biopsy specimens is useful for predicting the depth of invasion of early colorectal cancer: A Japanese collaborative study. J Gastroenterol 45: 1212-1218, 2010.

10. Okamoto Y, Fujimori T, Ohkura Y, Sugai T, Arai T, Watanabe G, Wada R, Ueno H, Togashi K, Yao T, et al: Histological assessment of intra- and inter-institutional reliabilities in detection of desmoplastic reaction in biopsy specimens of early colorectal carcinomas. Pathol Int 63: 539-545, 2013.

11. Kunz LA and Knuechel R: Tumor-associated fibroblasts (part I): Active stromal participants in tumor development and progression? Histol Histopathol 17: 599-621, 2002.

12. Angeli F, Koumakis G, Chen MC, Kumar S and Delinassios JG: Role of stromal fibroblasts in cancer: Promoting or impeding? Tumour Biol 30: 109-120, 2009.

13. Kudo SE, Sugihara Y, Kida H, Ishida F, Miyachi H, Mori Y, Misawa M, Hisayuki T, Kodama K, Wakamura K, et al: Depressed-type colonic lesions and 'de novo' cancer in familial adenomatous polyposis: A colonoscopist's viewpoint. ISRN Gastroenterol 2013: 838134, 2013.

14. Kimura R, Fujimori T, Ichikawa K, Ajioka Y, Ueno H, Ohkura Y, Kashida H, Togashi K, Yao T, Wada R, et al: Desmoplastic reaction in biopsy specimens of early colorectal cancer: A Japanese prospective multicenter study. Pathol Int 62: 525-531, 2012.

15. Landis JR and Koch GG: The measurement of observer agreement for categorical data. Biometrics 33: 159-174, 1977.

16. Worthley DL, Giraud AS and Wang TC: Stromal fibroblasts in digestive cancer. Cancer Microenviron 3: 117-125, 2010. 